\title{
Prevalência e fatores associados ao medo de cair em idosos sem histórico de quedas*
}

Prevalence and factors associated with fear of falling in elderly people with no history of falls

Prevalencia y factores asociados al miedo a las caídas en personas mayores sin antecedentes de caídas

\author{
Kátia Moreira da Silva', Rosemeiry Capriata de Souza Azevedo ${ }^{\text {II }}$, \\ Annelita Almeida de Oliveira Reiners ${ }^{\mathrm{III}}$, Adriana Delmondes de Oliveira ${ }^{\mathrm{IV}}$, \\ Ageo Mario Cândido da Silvav
}

\begin{abstract}
Resumo: Objetivo: analisar a prevalência e os fatores associados ao medo de cair em idosos sem histórico de quedas residentes na comunidade. Método: estudo transversal, realizado entre os meses de junho e agosto de 2018, com 140 pessoas de 65 anos ou mais sem histórico de quedas, cadastrados nas Estratégias de Saúde da Família. A variável dependente foi avaliada pela Falls Efficacy Scale - International - Brasil e as explicativas por questionário e instrumentos validados. Foram empreendidas análises descritiva, bivariada e o modelo de regressão múltipla de Poisson. Resultados: a prevalência do medo de cair foi de $65 \%$. Os fatores associados à variável dependente foram sintomas depressivos mais uso de polifarmácia e frequentar grupo social. Conclusão: a prevalência encontrada do medo de cair foi alta. Houve associação do desfecho com interação de polifarmácia e sintomas depressivos e a variável frequenta grupo social apresentou-se como fator de proteção.
\end{abstract}

Descritores: Idoso; Medo; Acidentes por Quedas; Enfermagem Geriátrica; Estudos Transversais

Abstract: Objective: to analyze the prevalence and factors associated with the fear of falling in communitydwelling elderly people with no history of falls. Method: a cross-sectional study, conducted between June and August 2018, with 140 people aged 65 years or older with no history of falls, registered in the Family Health Strategies. The dependent variable was assessed by the Falls Efficacy Scale - International - Brazil and the

\footnotetext{
${ }^{\text {I }}$ Enfermeira, mestra em enfermagem, Programa de Pós-Graduação em Enfermagem, Universidade Federal de Mato Grosso, Cuiabá, Mato Grosso, Brasil. E-mail: katiakawam@hotmail.com, Orcid: https://orcid.org/0000-0002-7679-0040

II Enfermeira, doutora em enfermagem, Programa de Pós-Graduação em Enfermagem, Universidade Federal de Mato Grosso, Cuiabá, Mato Grosso, Brasil. E-mail: rosemeirycapriataazevedo@gmail.com, Orcid: https://orcid.org/0000-0001-7986-5768

III Enfermeira, doutora em enfermagem, Programa de Pós-Graduação em Enfermagem, Universidade Federal de Mato Grosso, Cuiabá, Mato Grosso, Brasil. E-mail: annereiners.ar@gmail.com, Orcid: https://orcid.org/0000-0002-5699-8215

IV Enfermeira, mestra em enfermagem, Programa de Pós-Graduação em Enfermagem, Universidade Federal de Mato Grosso, Cuiabá, Mato Grosso, Brasil. E-mail: drydelmondes@gmail.com, Orcid: https://orcid.org/0000-0002-0100-413X

V Farmacêutico-bioquímico, doutor em Saúde Pública e Meio Ambiente, Programa de Pós-Graduação em Saúde Coletiva, Universidade Federal de Mato Grosso, Cuiabá, Mato Grosso, Brasil. E-mail: ageoms@hotmail.com, Orcid: https://orcid.org/0000-0001-5293-9413

* Extraído da dissertação "Fatores associados ao medo de cair em idosos sem histórico de quedas", Programa de Pós-Graduação em Enfermagem, Universidade Federal de Mato Grosso, 2019.
} 
explanatory variables by questionnaire and validated instruments. Descriptive and bivariate analyses were performed, as well as the Poisson multiple regression model. Results: the prevalence of fear of falling was $65 \%$. The factors associated with the dependent variable were depressive symptoms plus polypharmacy use and social group attendance. Conclusion: the prevalence found of fear of falling was high. There was an association of the outcome with the interaction of polypharmacy and depressive symptoms and the variable frequent social group presented as a protective factor.

Descriptors: Aged; Fear; Accidental Falls; Geriatric Nursing; Cross-Sectional Studies

Resumen: Objetivo: analizar la prevalencia y los factores asociados al miedo a las caídas en personas mayores sin antecedentes de caídas residentes en la comunidad. Método: estudio transversal, realizado entre junio y agosto de 2018, con 140 personas de 65 años o más sin antecedentes de caídas, registradas en las Estrategias de Salud de la Familia. La variable dependiente se evaluó mediante la Falls Efficacy Scale - International - Brasil y las variables explicativas mediante un cuestionario e instrumentos validados. Se realizaron análisis descriptivos, bivariados y el modelo de regresión múltiple de Poisson. Resultados: la prevalencia del miedo a caer fue del 65\%. Los factores asociados a la variable dependiente fueron los síntomas depresivos más el uso de polifarmacia y la asistencia a un grupo social. Conclusión: se encontró que la prevalencia del miedo a las caídas es alta. El resultado se asoció con la interacción de polifarmacia y síntomas depresivos, y la variable grupo social frecuentado fue un factor protector.

Descriptores: Anciano; Miedo; Accidentes por Caídas; Enfermería Geriátrica; Estudios Transversales

Descriptores: Anciano; Miedo; Accidentes por Caídas; Enfermería Geriátrica; Estudios Transversales

\section{Introdução}

O medo de cair (MC) é uma das ameaças mais importantes para a independência e autonomia dos idosos, pois acarreta mudanças físicas, funcionais e psicossociais, com impactos negativos na vida desses indivíduos e profissionais de saúde, somado ao aumento na demanda e custos assistenciais. ${ }^{1-2}$

Está associado a desfechos negativos a saúde, iniciando uma espiral de eventos debilitantes que podem culminar em quedas, declínio funcional, institucionalização e hospitalização, impactando negativamente na qualidade de vida dos idosos. ${ }^{1,3-4}$ É um fenômeno presente na vida dos idosos e pode persistir em uma parcela significativa deles, independentemente do histórico de quedas. ${ }^{5-6}$

Embora se tenha conhecimento sobre a prevalência do MC em idosos sem histórico de quedas, estudos têm apresentado essas frequências nesta população, apenas como resultado secundário ${ }^{7-8}$ e os fatores que podem levar esses idosos a desenvolver o MC ainda não estão esclarecidos. 
Estudo de revisão sistemática que investigou a prevalência do MC entre idosos caidores e não caidores mostrou que o MC entre não caidores é superior a 50\%.7 Outro estudo de revisão de literatura, realizado nos Estados Unidos apresentou uma prevalência do MC de $12 \%$ a $65 \%$ nos idosos não caidores. ${ }^{8}$

No que diz respeito às quedas, a associação entre o MC e esse evento está bem estabelecida. Existe uma relação bidirecional na qual as pessoas mais velhas estão mais propensas a cair e a partir desse momento podem desenvolver o MC. Igualmente, sabe-se que tanto o MC quanto as quedas resultam de fatores de risco comuns, como ter idade avançada, pertencer ao sexo feminino, apresentar distúrbios do equilíbrio e da marcha, apresentar sintomas depressivos, déficit de mobilidade, restrição de atividades e redução das atividades sociais. ${ }^{3,9}$

Até o momento, não foram encontrados estudos com objetivo de verificar os fatores associados ao MC exclusivamente nos idosos sem histórico de quedas, o que torna relevante conhecer as particularidades desse fenômeno nesta população. Diante do exposto, surgiu o questionamento: que fatores estão associados ao $\mathrm{MC}$ em idosos sem histórico de quedas residentes na comunidade?

A identificação dos fatores associados ao MC nos idosos que nunca caíram é importante para ampliar o conhecimento sobre o fenômeno e planejar ações de cuidado que possam prevenir e/ou minimizar o MC nesses indivíduos. O estudo teve por objetivo analisar a prevalência e os fatores associados ao MC em idosos sem histórico de quedas residentes na comunidade.

\section{Método}

Estudo transversal, de amostragem probabilística, empreendido com pessoas de 65 anos ou mais, cadastradas em 11 unidades de Estratégia de Saúde da Família (ESF) localizadas na região Norte da cidade de Cuiabá, Mato Grosso, Brasil. 
Prevalência e fatores associados ao medo de cair em idosos sem histórico de quedas $\mid 4$

O cálculo amostral foi realizado partindo-se do universo de 1804 idosos com 65 anos ou mais, cadastrados nas ESF, com base em uma população finita, nível de confiança de 95\%, erro de amostragem de $\pm 8 \%$. Diante da variação de $12 \%$ a $65 \%$ na proporção de idosos com $\mathrm{MC}$ sem histórico de quedas encontrada na literatura, ${ }^{8}$ e não se encontrando valores mais exatos na literatura brasileira, adotou-se a mediana de $38 \%$ de modo o valor mais parcimonioso para a amostragem, sendo esta prevalência a mais representativa para o presente estudo. A amostra mínima necessária para realização desse estudo foi de 131 idosos, no entanto, foram incluídos mais nove idosos que manifestaram o desejo em participar da pesquisa no decorrer da coleta de dados, perfazendo o total de 140 participantes.

Foram incluídos no estudo pessoas com idade igual ou maior que 65 anos, cadastrados nas ESF e residentes na área urbana, que deambulasse na posição ortostática, com ou sem dispositivo de auxílio a marcha, com capacidade cognitiva e de comunicação preservados avaliados pelo Mini Exame do Estado Mental (MEEM). ${ }^{10}$ Foram excluídos idosos acamados, cadeirantes, que referiram ter caído pelo menos uma vez após ter completado 60 anos, com sequelas de acidente vascular encefálico, deficiência auditiva e visual, acometidos por doença de Parkinson e Alzheimer, bem como outros déficits de mobilidade e/ou vestibulares referidos pelo próprio idoso ou seu cuidador. Idosos não encontrados após três tentativas de busca ou, nos casos de óbito foram substituídos por meio de novo sorteio.

A coleta de dados foi realizada entre os meses de junho e agosto de 2018 por meio de entrevista estruturada no domicílio dos idosos, pela pesquisadora principal acompanhada por agentes comunitários de saúde, após assinatura do Termo de Consentimento Livre e Esclarecido, o que garantiu que garantiu que não houvesse dados faltantes referentes às variáveis de interesse.

Para caracterização das variáveis independentes foram utilizados questionário estruturado de dados sociodemográficos e de condições de saúde e instrumentos de avaliação validados no Brasil. As variáveis independentes estudadas foram faixa etária, sexo, estado civil, 
5 | Silva KM, Azevedo RCS, Reiners AAO, Oliveira AD, Silva AMC

situação ocupacional, anos de estudo, arranjo familiar e renda, autoavaliação de saúde, problema de saúde autorreferido e quantidade de problemas, alterações de visão e audição autorreferidos, uso contínuo de medicamentos, principais medicamentos, polifarmácia - definida como uso concomitante de cinco ou mais medicamentos ${ }^{11}$ prescritos ou não, pratica atividade física, capacidade funcional, mobilidade, humor e condição nutricional realizada por meio de medidas antropométricas, classificada em baixo peso (IMC $\leq 22)$, eutrófico $(\mathrm{IMC}>22$ e $<27)$ e sobrepeso $(\mathrm{IMC} \geq 27) .{ }^{12}$

Também se utilizou instrumentos validados como a escala de $\mathrm{Katz}^{13}$ para avaliar as habilidades funcionais de idosos no desempenho das atividades de vida diária (AVD). Foi classificado como independente o idoso que referiu realizar todas as atividades de forma independente sem necessidade de ajuda e classificado como dependente àquele que referiu necessitar de ajuda para realização de pelo menos uma das atividades.

Para avaliar as habilidades funcionais dos idosos na realização das atividades instrumentais da vida diária (AIVD) foi utilizada a escala de Lawton e Brody (escore de 9 a 27 pontos). ${ }^{14}$ Foi classificado como independente o idoso que apresentou escore $\geq 19$ pontos e classificado como dependente àquele que apresentou escore $\leq 18$ pontos.

A mobilidade foi avaliada por meio do Timed Up and Go test, que avalia o tempo (em segundos) que o idoso leva para se levantar de uma cadeira onde está sentado com as costas apoiadas, levanta-se, caminha três metros, vira, volta rumo à cadeira e senta-se novamente. ${ }^{15}$ Neste estudo foi classificado normal (sem déficit de mobilidade) o idoso que realizou o teste no tempo de até 10 segundos e com déficit de mobilidade àquele que realizou o teste no tempo $\geq 11$ segundos.

A variável humor foi avaliada por meio da escala de Depressão Geriátrica Abreviada (Geriatric Depression Scale - GDS-15), composta por 15 perguntas na qual o escore pode variar de 0 a 15 pontos. ${ }^{16}$ O humor foi classificado em normal ( 0 a 5 pontos) e sintomas depressivos ( 6 a 15 pontos).

A variável desfecho, MC foi obtida por meio da Falls Efficacy Scale-International- Brasil (FES-I-BRASIL)..$^{1,5,17-19}$ Este instrumento é constituído por 16 questões, pontuadas em uma escala 
Prevalência e fatores associados ao medo de cair em idosos sem histórico de quedas $\mid 6$

tipo Likert de 1 a 4, na qual os participantes identificam a preocupação com a possibilidade de cair durante a realização de AVD, AIVD e participação social, imaginando como normalmente desenvolve a atividade e, se atualmente não a faz, responder como se tivesse que realizar tal atividade. O escore total é obtido pela soma dos pontos que pode variar de 16 a 64 pontos e permite avaliar os graus de MC. Foram classificados como não possui MC idosos com escore de 16 a 22 pontos e possui MC idosos com escore de 23 a 64 pontos. ${ }^{18-19}$

Os dados foram gerenciados por meio do software STATA versão 12.0. Na análise descritiva as variáveis foram descritas em frequências absoluta (n) e relativa (\%). Na análise bivariada, foram identificadas as associações entre a variável desfecho (MC) e as demais variáveis de exposição por meio do cálculo da razão de prevalência (RP) utilizando-se o método Qui-quadrado de Mantel-Haenszel ou teste exato de Fischer quando necessário, com intervalo de confiança de 95\%. Foram realizadas duas análises estratificadas: a primeira, estratificando-se a comparação entre sexo e MC pela faixa etária, na segunda, utilizou-se na estratificação das análises as variáveis uso de polifarmácia na comparação entre presença de sintomas depressivos e MC. As variáveis que apresentaram $\mathrm{p} \leq 0,20$ foram selecionadas para análise múltipla por meio da Regressão de Poisson, utilizando-se o método hierárquico de inclusão de variáveis. No modelo final, por meio da Regressão de Poisson para avaliação de potenciais efeitos confundidores não aferidos, foram mantidas as associações que apresentaram nível de significância menor que 0,05 .

O estudo foi aprovado pelo Comitê de Ética em Pesquisa da Universidade Federal de Mato Grosso, sob parecer de n²0666.399 de 21/05/2018. Todos os preceitos éticos que envolve pesquisa com seres humanos foram respeitados. Esta pesquisa faz parte do estudo multicêntrico "Envelhecimento ativo: promoção da saúde e prevenção de incapacidade funcional e cognitiva" (PROCAD Edital nº 071/2013) realizado com o apoio da Coordenação de Aperfeiçoamento de Pessoal de Nível Superior - Brasil (CAPES). 
7 | Silva KM, Azevedo RCS, Reiners AAO, Oliveira AD, Silva AMC

\section{Resultados}

Participaram deste estudo 140 idosos. A prevalência do MC nos idosos sem histórico de quedas é de 65\% (IC: 56,49 - 72,86), com média do escore total da FES-I-BRASIL na população avaliada de 26,85 pontos.

Dos 91 idosos com MC, a maioria $(64,8 \%)$ é do sexo feminino, casada $(57,1 \%)$ e nunca estudou (58,2\%). Grande parte (46,1\%) está na faixa etária de 70 a 79 anos, com média de idade de 70,95 anos $(\mathrm{DP} \pm 5,27)$. Houve predominância de idosos aposentados $(83,5 \%)$, com renda de até um salário mínimo (73,6\%). A maioria dos idosos (53,8\%) frequenta grupos sociais como igreja e centro de convivência.

Em relação às condições de saúde, a maioria dos idosos $(86,8 \%)$ refere ter dois ou mais problemas de saúde, dos quais os mais frequentes são problemas de visão (76,9\%) e a Hipertensão Arterial Sistêmica (73,6\%). A maioria (72,5\%) dos idosos é independente para realização das atividades básicas e instrumentais de vida diária $(69,2 \%)$. Quanto à percepção de saúde 52,7\% autoavaliam como regular, 54,9\% faz uso contínuo de até quatro medicamentos, principalmente os anti-hipertensivos (72,5\%). A maioria $(84,6 \%)$ não pratica atividade física, grande parte (45\%) está com sobrepeso, e 52,7\% apresenta sintomas depressivos.

$\mathrm{Na}$ análise bivariada as variáveis sociodemográficas que se mostraram associadas ao MC foram: sexo feminino ( $\mathrm{RP}=1,42$ IC 95\% 1,08-1,86), idade igual ou superior a 70 anos $(\mathrm{RP}=1,18$ IC $95 \% 0,92-1,51)$, aposentado ( $\mathrm{RP}=2,09$ IC 95\% 1,07-4,05) e frequenta grupo social ( $\mathrm{RP}=0,70$ IC 95\% 0,55-0,89), sendo a última um fator de proteção e os demais fatores de risco para o MC (Tabela 1). 
Prevalência e fatores associados ao medo de cair em idosos sem histórico de quedas $\mid 8$

Tabela 1 - Prevalência e Razão de prevalência do MC em idosos que nunca caíram segundo características sociodemográficas. Cuiabá-MT, 2018.

\begin{tabular}{|c|c|c|c|c|}
\hline \multirow{2}{*}{ Variáveis } & \multicolumn{4}{|c|}{ Medo de cair } \\
\hline & $\mathrm{n} / \mathrm{N}^{*}$ & $\%$ & $\mathrm{RP}^{+}$bruta $\left(\mathrm{IC}^{*} 95 \%\right)$ & Valor de $\mathrm{p}^{\S}$ \\
\hline \multicolumn{5}{|l|}{ Sexo } \\
\hline Masculino & $32 / 61$ & 52,5 & 1 & \\
\hline Feminino & $59 / 79$ & 74,7 & $1,42(1,08-1,86)$ & 0,006 \\
\hline \multicolumn{5}{|l|}{ Faixa etária } \\
\hline $65-69$ anos & $41 / 69$ & 59,4 & 1 & \\
\hline 70 anos e mais & $50 / 71$ & 70,4 & $1,18(0,92-1,51)$ & 0,173 \\
\hline \multicolumn{5}{|l|}{ Estado civil } \\
\hline Com companheiro(a) & $52 / 81$ & 64,2 & 1 & \\
\hline Sem companheiro(a) & $39 / 59$ & 66,1 & $1,02(0,80-1,31)$ & 0,816 \\
\hline \multicolumn{5}{|l|}{ Escolaridade em anos } \\
\hline 4 anos e mais de estudo & $26 / 37$ & 70,3 & 1 & \\
\hline 0 a 3 anos & $65 / 103$ & 63,1 & $0,89(0,69-1,16)$ & 0,434 \\
\hline \multicolumn{5}{|l|}{ Arranjo familiar } \\
\hline Acompanhado(a) & $75 / 117$ & 64,1 & 1 & \\
\hline Sozinho(a) & $16 / 23$ & 69,6 & $1,08(0,80-1,46)$ & 0,616 \\
\hline \multicolumn{5}{|l|}{ Situação ocupacional } \\
\hline Trabalhando (a) & $6 / 18$ & 33,3 & 1 & \\
\hline Aposentado(a) & $85 / 122$ & 69,7 & $2,09(1,07-4,05)$ & 0,002 \\
\hline \multicolumn{5}{|l|}{ Renda per capita } \\
\hline Mais de $1 \mathrm{SM}$ & $15 / 26$ & 57,7 & 1 & \\
\hline 0 a $1 \mathrm{SM}$ & $76 / 114$ & 66,7 & $1,15(0,81-1,64)$ & 0,388 \\
\hline \multicolumn{5}{|l|}{ Frequenta grupo social } \\
\hline $\operatorname{Sim}$ & $49 / 63$ & 77,8 & $0,70(0,55-0,89)$ & 0,004 \\
\hline Não & $42 / 77$ & 54,5 & 1 & \\
\hline \multicolumn{5}{|l|}{ Pratica atividade física } \\
\hline $\operatorname{Sim}$ & $14 / 22$ & 63,6 & 1 & \\
\hline Não & $77 / 118$ & 65,2 & $1,02(0,72-1,44)$ & 0,884 \\
\hline
\end{tabular}

$\mathrm{N}^{*}=140 ; \mathrm{R} \mathrm{P}^{\dagger}$ : Razão de Prevalência; $\mathrm{IC}^{+}$95\%: intervalo de confiança para a proporção de 95\%; $\mathrm{p}^{\text {s: }}$ Nível de significância considerando a distribuição de Qui-Quadrado (valor de $\mathrm{p} \leq 0,05$ ). SM - salário mínimo.

Em relação a análise bivariada das variáveis de condições de saúde e sua relação com o MC, verificou-se associação estatisticamente significativas com: alterações de audição ( $R P=1,21$ IC $95 \%$ 0,93 - 1,57), polifarmácia ( $\mathrm{RP}=1,21$ IC $95 \% 0,95-1,54)$, baixo peso/sobrepeso ( $\mathrm{RP}=1,34$ IC 
9 | Silva KM, Azevedo RCS, Reiners AAO, Oliveira AD, Silva AMC

95\% 1,01-1,77), déficit mobilidade ( $\mathrm{RP}=1,20$ IC 95\% 0,92-1,56) e sintomas depressivos $(\mathrm{RP}=1,32$ IC 95\% 1,04-1,68) (Tabela 2).

Tabela 2 - Prevalência e Razão de prevalência do MC em idosos que nunca caíram segundo condições de saúde. Cuiabá-MT, 2018.

\begin{tabular}{|c|c|c|c|c|}
\hline \multirow{2}{*}{ Variáveis } & \multicolumn{4}{|c|}{ Medo de cair } \\
\hline & $\mathrm{n} / \mathrm{N}^{*}$ & $\%$ & $\mathrm{RP}^{+}$bruta $\left(\mathrm{IC}^{*} 95 \%\right)$ & Valor de $\mathrm{p}^{\S}$ \\
\hline \multicolumn{5}{|l|}{ Autoavaliação de saúde } \\
\hline Positiva & $29 / 40$ & 72,5 & 1 & \\
\hline Negativa & $62 / 100$ & 62 & $0,85(0,66-1,09)$ & 0,241 \\
\hline \multicolumn{5}{|l|}{ Problema de saúde referido } \\
\hline Não & $5 / 6$ & 83,3 & 1 & \\
\hline $\operatorname{Sim}$ & $86 / 134$ & 64,2 & $0,77(0,52-1,12)$ & 0,337 \\
\hline \multicolumn{5}{|l|}{ Número de problemas de saúde } \\
\hline Até 1 problema & $12 / 16$ & 75 & 1 & \\
\hline 2 ou mais & $79 / 124$ & 63,7 & $0,84(0,62-1,16)$ & 0,374 \\
\hline \multicolumn{5}{|l|}{ Alterações de saúde referidas } \\
\hline \multicolumn{5}{|l|}{ Visão } \\
\hline Não & $21 / 33$ & 63,6 & 1 & \\
\hline $\operatorname{Sim}$ & $70 / 107$ & 65,4 & $1,02(0,76-1,37)$ & 0,851 \\
\hline \multicolumn{5}{|l|}{ Audição } \\
\hline Não & $72 / 115$ & 62,6 & 1 & \\
\hline $\operatorname{Sim}$ & $19 / 25$ & 76 & $1,21(0,93-1,57)$ & 0,204 \\
\hline \multicolumn{5}{|l|}{ Uso de medicamentos } \\
\hline Não & $12 / 17$ & 70,5 & 1 & \\
\hline $\operatorname{Sim}$ & $79 / 123$ & 64,2 & $0,90(0,65-1,27)$ & 0,607 \\
\hline \multicolumn{5}{|l|}{ Polifarmácia } \\
\hline Não & $62 / 101$ & 61,4 & 1 & \\
\hline $\operatorname{Sim}$ & $29 / 39$ & 74,4 & $1,21(0,95-1,54)$ & 0,150 \\
\hline \multicolumn{5}{|c|}{ Atividades básicas de vida diária (Katz) } \\
\hline Independente & $66 / 105$ & 62,9 & 1 & \\
\hline Dependente & $25 / 35$ & 71,4 & $1,13(0,87-1,46)$ & 0,358 \\
\hline \multicolumn{5}{|c|}{ Atividades instrumentais de vida diária (L\&B) } \\
\hline Independente & $63 / 99$ & 63,6 & 1 & \\
\hline Dependente & $28 / 41$ & 68,3 & $1,07(0,83-1,38)$ & 0,600 \\
\hline \multicolumn{5}{|l|}{ Índice de massa corporal (IMC) } \\
\hline Eutrófico & $29 / 54$ & 53,7 & 1 & \\
\hline Baixo peso/sobrepeso & $62 / 86$ & 72,1 & $1,34(1,01-1,37)$ & 0,026 \\
\hline \multicolumn{5}{|l|}{ Mobilidade (TUG) } \\
\hline Normal & $33 / 57$ & 57,9 & 1 & \\
\hline Déficit mobilidade & $58 / 83$ & 69,9 & $1,20(0,92-1,56)$ & 0,145 \\
\hline \multicolumn{5}{|l|}{ Humor (GDS) } \\
\hline Normal & $43 / 76$ & 56,6 & 1 & \\
\hline
\end{tabular}


Prevalência e fatores associados ao medo de cair em idosos sem histórico de quedas | 10

Sintomas depressivos

$48 / 64 \quad 75 \quad 1,32(1,04-1,68)$

0,023

$\mathrm{N}^{*}=140$; RP: Razão de Prevalência; IC ${ }^{*}$ 95\%: intervalo de confiança para a proporção de 95\%; Ps: Nível de significância considerando a distribuição de Qui-Quadrado (valor de $\mathrm{p} \leq 0,05$ ).

Ao se estratificar a comparação entre sexo e MC pelas faixas etárias dicotomizadas em 65 a 69 e 70 anos e mais, em ambas houve associações estatisticamente significantes. Contudo, nota-se que entre os idosos mais jovens indivíduos do sexo feminino apresentaram maior associação com MC em relação aos homens, em relação à mesma comparação entre os mais velhos (Tabela 3).

Tabela 3 - Razão de prevalência e intervalo de confiança (IC 95\%) de sexo e MC estratificada por faixa etária. Cuiabá, MT, Brasil, 2018.

\begin{tabular}{cccccccc}
\hline Variável & \multicolumn{2}{c}{ MC } & \multicolumn{2}{c}{ Sem MC } & $\mathbf{R P}^{+}$ & IC $^{*}$ & Valor de $\mathbf{p}^{\S}$ \\
\hline $\mathbf{6 5}$ a $\mathbf{6 9}$ anos & $\mathbf{n}^{*}$ & $\%$ & $\mathbf{n}^{*}$ & $\%$ & & & \\
Feminino & 31 & 68,89 & 14 & 31,11 & 1,65 & $1,03-2,76$ & $\mathbf{0 , 0 2 9}$ \\
Masculino & 10 & 41,67 & 14 & 58,33 & & 1 & \\
70 anos e mais & & & & & & & \\
Feminino & 28 & 82,35 & 6 & 17,65 & 1,38 & $1,01-1,88$ & $\mathbf{0 , 0 3 6}$ \\
Masculino & 22 & 59,46 & 15 & 40,54 & & 1 &
\end{tabular}

$\mathrm{N}^{*}=140$; RP: Razão de Prevalência; $\mathrm{IC}^{+}$95\%: intervalo de confiança para a proporção de 95\%; P\$: Nível de significância considerando a distribuição de Qui-Quadrado (valor de $\mathrm{p} \leq 0,05$ ).

Na comparação entre presença de sintomas depressivos e MC, estratificado pelo uso de polifarmácia, apenas entre os que fazem uso de polifarmácia houve essa associação (Tabela 4).

Tabela 4 - Razão de prevalência e intervalo de confiança (IC 95\%) de sintomas depressivos e MC estratificada por uso de polifarmácia. Cuiabá, MT, Brasil, 2018.

\begin{tabular}{|c|c|c|c|c|c|c|c|}
\hline \multirow{2}{*}{ Variável } & \multicolumn{2}{|c|}{$\mathrm{MC}$} & \multicolumn{2}{|c|}{ Sem MC } & \multirow{2}{*}{$\mathbf{R P}^{+}$} & \multirow{2}{*}{$\mathrm{IC}^{*}$} & \multirow{2}{*}{ Valor de $\mathrm{p}^{\S}$} \\
\hline & $\mathrm{n}^{*}$ & $\%$ & $\mathrm{n}^{*}$ & $\%$ & & & \\
\hline \multicolumn{8}{|l|}{ Não faz uso de polifarmácia } \\
\hline Presença de sintomas depressivos & 26 & 66,7 & 13 & 33,3 & 1,14 & $0,84-1,56$ & 0,389 \\
\hline Ausência de sintomas depressivos & 36 & 58,1 & 26 & 41,9 & & 1 & \\
\hline \multicolumn{8}{|l|}{ Uso de polifarmácia } \\
\hline Presença de sintomas depressivos & 22 & 88 & 3 & 12 & 1,76 & $1,02-3,03$ & 0,019 \\
\hline Ausência de sintomas depressivos & 7 & 50 & 7 & 50 & & 1 & \\
\hline
\end{tabular}


Ao final, para análise múltipla, utilizando o modelo hierárquico da Regressão de Poisson, foram incluídas todas as variáveis com $\mathrm{p} \leq 0,20$. Delas permaneceram associadas ao $\mathrm{MC}$ as variáveis frequenta grupo social e uso de polifarmácia com sintomas depressivos (Tabela 5).

Tabela 5 - Análise do modelo final da Regressão Múltipla de Poisson para variáveis associadas ao MC em idosos que nunca caíram. Cuiabá, MT, Brasil, 2018.

\begin{tabular}{lcc}
\hline \multicolumn{1}{c}{ Variáveis } & RP $^{*}($ IC'95\%) & Valor de $\mathbf{p}^{*}$ \\
\hline Frequenta grupo social & $0,74(0,58-0,93)$ & $\mathbf{0 , 0 2 1}$ \\
Polifarmácia e sintomas depressivos & $1,37(1,12-1,67)$ & $\mathbf{0 , 0 0 2}$
\end{tabular}

RP*: Razão de Prevalência; IC 95\%: intervalo de confiança para a proporção de 95\%; P*: Nível de significância considerando a distribuição de Qui-Quadrado (valor de p<0,05). Ajustado pela variável sexo.

\section{Discussão}

Trata-se de pesquisa pioneira pois, é um dos primeiros estudos que investigou especificamente os fatores associados ao MC em idosos sem histórico de quedas residentes na comunidade. A alta prevalência do $\mathrm{MC}$ em idosos encontrada nesta pesquisa é um achado que não surpreende e pode estar relacionado à idade mais avançada dos participantes. Sabe-se que, na medida em que a idade avança, mudanças próprias do envelhecimento podem influenciar a avaliação do idoso quanto ao seu risco de cair, tais como declínio físico-funcional e diminuição das reservas fisiológicas, alterações nos sistemas musculoesquelético, neuromuscular e sensorial. ${ }^{5}$ Além disso, o surgimento de novas condições crônicas como osteoporose e doenças musculoesqueléticas, fraqueza, dores, desequilíbrio e dificuldades na locomoção, ${ }^{1,20}$ também podem levar a um maior MC.

Outra explicação para esse resultado pode ser pelo fato de que a maioria dos participantes é do sexo feminino, pois além das comorbidades, a baixa densidade óssea e a perda da massa muscular devido à diminuição hormonal, ${ }^{1}$ tornam sua estrutura óssea e muscular mais frágeis em comparação aos homens. ${ }^{21}$ Além disso, no que se refere à saúde, as mulheres apresentam maior capacidade de identificar os riscos. ${ }^{1}$ 
Prevalência e fatores associados ao medo de cair em idosos sem histórico de quedas | 12

A maioria das mulheres participantes do estudo foram classificadas como independentes tanto para atividades básicas quanto para as atividades instrumentais de vida diária. É possível que, por ter conhecimento sobre os riscos e consequências das quedas a que estão expostas na realização daquelas atividades, as mulheres idosas desenvolvam o MC, mesmo sem nunca ter caído.

Neste estudo, nos idosos do sexo masculino da faixa etária de 65 a 69 anos o MC é menor que nos da faixa etária de 70 anos e mais. Esse achado pode ser explicado pelo fato de que homens idosos mais jovens, apesar do envelhecimento, tendem a superestimar suas capacidades físicas e subestimar suas limitações funcionais. ${ }^{22}$ Contudo, o MC entre os homens mais velhos se aproxima do encontrado nas mulheres mais velhas. Provavelmente, a razão desse resultado seja porque os homens nessa faixa etária apresentam maiores limitações, visto que as alterações decorrentes do envelhecimento se acumulam progressivamente, o que pode contribuir para o desenvolvimento do MC na idade mais avançada. ${ }^{5,17}$

As variáveis comumente associadas ao MC como dependência para realização de AVD e AIVD, déficit de mobilidade, autopercepção negativa de saúde, idade avançada e sexo feminino, não estiveram associadas ao MC nesse estudo. Uma possível explicação é que idosos com histórico de quedas podem apresentar características distintas dos idosos que nunca caíram.

Um dos principais resultados deste estudo é a associação entre $\mathrm{MC}$ e presença de sintomas depressivos mais uso de polifarmácia. Ou seja, quando as pessoas que têm sintomas depressivos fazem uso de polifarmácia, o risco de desenvolver o MC é maior.

A associação entre MC e os sintomas depressivos, é um achado apoiado por outros resultados encontrados na literatura. ${ }^{22-23}$ É possível que a presença de sintomas depressivos constitua um fator de risco para o desenvolvimento do $\mathrm{MC}$, uma vez que podem comprometer a marcha e o equilíbrio, na medida em que são frequentemente acompanhados de cansaço e diminuição de energia, interferindo na estabilidade do corpo e nas habilidades funcionais, tornando os idosos menos seguros e confiantes em suas habilidades físicas levando-os a ter MC..$^{22-23}$ 
O tratamento farmacológico destinado aos sintomas depressivos como ansiolíticos, antidepressivos e antipsicóticos, pode ter efeitos sobre o sistema nervoso central que alteram aspectos cognitivos e psicomotores do organismo e seus principais efeitos podem ser a sedação, hipnose e relaxamento muscular. ${ }^{24}$

No caso dos idosos, as características do envelhecimento como redução das reservas funcionais e alterações fisiológicas podem alterar tanto a farmacocinética quanto a farmacodinâmica, podendo resultar na redução ou aumento da biodisponibilidade do fármaco devido a alterações na absorção, metabolismo, distribuição e eliminação das drogas. ${ }^{24-25}$ Quando somadas à polifarmácia podem contribuir para ocorrência de problemas relacionados aos medicamentos como acentuação dos sintomas depressivos, interações medicamentosas e reações adversas. ${ }^{26}$

Ocorrências iatrogênicas decorrentes de medicamentos podem levar a alteração no equilíbrio, hipotensão postural, sonolência, diminuição dos reflexos, tonturas, bradicardia, fadiga e necessidade de urinar com maior frequência. ${ }^{10,24} \mathrm{Na}$ medida em que esses fatores ocorrem ao mesmo tempo, pode aumentar a chance de os idosos desenvolverem o MC.

Neste estudo, frequentar grupos sociais foi encontrado como fator de proteção ao MC. Essa associação foi identificada em um estudo prévio realizado com idosos. ${ }^{27}$ Provavelmente isso ocorre porque os idosos nos grupos sociais, estabelecem relações que proporcionam encontros com pessoas com condições semelhantes. Esses encontros lhes permitem a troca de informações e a construção de saberes significativos ao seu cotidiano ${ }^{28}$ favorecendo o conhecimento sobre as quedas e as consequências que seus pares tiveram.

Embora o contexto atual de Pandemia provocado pela Covid-19 exija medidas preventivas como distanciamento social, a participação em grupos também permite que os idosos estabeleçam novos vínculos e amizades, que contribuem para melhor qualidade de vida. ${ }^{28}$ Além disso, os grupos proporcionam práticas de autocuidado por meio de atividades físicas que retiram o idoso da condição de sedentarismo, influenciando na sua saúde física e psicológica. ${ }^{29}$ 
Prevalência e fatores associados ao medo de cair em idosos sem histórico de quedas | 14

O envolvimento comunitário pode ser um fator significativo para melhorar a percepção da confiança pessoal, pois pode promover bem-estar físico, cognitivo e psicológico ${ }^{30}$ e contribuir para redução da ocorrência do MC. Essa participação poderá ser retomada pelos idosos na medida em que a população seja vacinada e que a disseminação do vírus esteja reduzida.

Pode-se apontar como limitação o fato de as informações serem obtidas pelo autorrelato, podendo haver subestimação de prevalências de algumas condições de saúde, bem como a possibilidade da ocorrência de outros vieses, em especial o de memória. Contudo, a utilização da FES-I-BRASIL, considerada padrão ouro para o estudo desse fenômeno, permite que os resultados sejam comparáveis com outras publicações nacionais e internacionais que estudam o MC.

Os resultados deste estudo indicam que os profissionais de saúde devem incluir na avaliação geriátrica a identificação do MC nos idosos que nunca caíram, na medida em que esse fenômeno pode trazer impactos negativos na vida desses idosos, colocando em risco sua autonomia e independência. Também podem subsidiar o planejamento de ações que visam à promoção da saúde, prevenção e redução do MC nos idosos, esclarecendo dúvidas sobre o MC e as repercussões que pode gerar na vida dos idosos, familiares e cuidadores, principalmente naqueles com fatores de risco modificáveis como polifarmácia e sintomas depressivos.

\section{Conclusão}

A prevalência do MC em idosos sem histórico de quedas encontrada foi alta. A presença concomitante de polifarmácia e referência de sintomas depressivos foi preditora para o MC. A participação de grupos sociais se associou inversamente à referência de MC e se apresentou como fator de proteção.

Estes resultados são importantes pois demostram a importância da avaliação do MC em todos os idosos, a fim de se identificar precocemente essa condição. Isso impacta diretamente na prática clínica dos profissionais de saúde, visto que esse fenômeno acarreta alterações físicas, funcionais e psicoemocionais na vida das pessoas mais velhas. Além disso, aponta para a 
necessidade de planejar ações de prevenção de quedas e estabelecer estratégias apropriadas para garantir o envelhecimento saudável.

Sugere-se que novos estudos acerca do MC sejam realizados com a população idosa sem histórico de quedas a fim de comparar com o resultado apresentado, bem como, para verificação da efetividade de intervenções para redução do MC nestes idosos.

\section{Referências}

1. Vitorino LM, Teixeira CAB, Boas ELV, Pereira RL, Santos NO, Rozendo CA. Fear of falling in older adults living at home: associated factors. Rev Esc Enferm USP. 2017;51:e03215. doi: 10.1590/S1980-220X2016223703215

2. Pena SB, Guimarães HCQCP, Lopes JL, Guandalini LS, Taminato M, Barbosa DA, et al. Fear of falling and risk of falling: a systematic review and meta-analysis. Acta Paul Enferm. 2019;32(4):456-63. doi: 10.1590/1982-0194201900062

3. Park JI, Yang JC, Chung S. Risk factors associated with the fear of falling in community-living elderly people in Korea: role of psychological factors. Psychiatry Investig. 2017;14(6):894-9. doi:10.4306/pi.2017.14.6.894

4. Choi K, Jeon GS, Cho SI. Prospective study on the impact of fear of falling on functional decline among community dwelling elderly women. Int J Environ Res Public Health. 2017;14(5):469. doi:10.3390/ijerph14050469

5. Cruz DT, Duque RO, Leite ICG. Prevalence of fear of falling, in a sample of elderly adults in the community. Rev Bras Geriatr Gerontol. 2017;20(3):309-18. doi: 10.1590/1981-22562017020.160176

6. Makino K, Makizako H, Doi T, Tsutsumimoto K, Hotta R, Nakakubo S, et al. Fear of falling and gait parameters in older adults with and without fall history. Geriatr Gerontol Int. 2017;17(12):2455-9. doi: 10.1111/ggi.13102

7. Scheffer AC, Schuurmans MJ, Van Dijk N, Van der Hooft T, De Rooij SE. Fear of falling: measurement strategy, prevalence, risk factors and consequences among older persons. Age Ageing. 2008 Jan;37(1):19-24. doi: 10.1093/ageing/afm169

8. Legters K. Fear of falling. Phys Ther. 2002;82(3):264-72. doi: 10.1093/ptj/82.3.264

9. Bittencourt VLL, Graube SL, Stumm EMF, Battisti IDE, Loro MM, Winkelmann ER. Factors associated with the risk of falls in hospitalized adult patients. Rev Esc Enferm USP. 2017;51:e03237. doi: 10.1590/S1980-220X2016037403237

10. Bertolucci PHF, Brucki SMD, Campacci SR, Juliano Y. O Mini-exame do estado mental em uma população geral: impacto da escolaridade. Arq Neuropsiquiatr [Internet]. 1994 [acesso em $2020 \mathrm{dez}$ 10];52(1):01-7. Disponívek em: https://www.scielo.br/pdf/anp/v52n1/01.pdf

11. Marques GFM, Rezende DMRP, Silva IP, Souza PC, Barbosa SRM, Penha RM, et al. Polypharmacy and potentially inappropriate medications for elder people in gerontological nursing. Rev Bras Enferm. 2018;71(5):2440-6. doi: 10.1590/0034-7167-2017-0211

12. Ministério da Saúde (BR), Secretaria de Atenção a Saúde. Envelhecimento e saúde da pessoa idosa. Brasília (DF): Ministério da Saúde; 2007. 
Prevalência e fatores associados ao medo de cair em idosos sem histórico de quedas | 16

13. Katz S, Downs TD, Cash HR, Grotz RC. Progress in development of the index of ADL. Gerontologist. 1970 Spring;10(1):20-30. doi: 10.1093/geront/10.1_part_1.20

14. Lawton M, Brody E. Assessment of older people: selfmaintaining and instrumental activities of daily living. Gerontologist. 1969 Autumn;9(3):179-86. doi: 10.1093/geront/9.3_Part_1.179

15. Bischoff HA, Stähelin HB, Monsch AU, Iversen MD, Weyh A, Von Dechend M, et al. Identifying a cut-off point for normal mobility: a comparison of the timed "up and go" test in community-dwelling and institutionalised elderly women. Age Ageing. 2003 May;32(3):315-20. doi: 10.1093/ageing/32.3.315

16. Yesavage JA, Brink TL, Rose TL, Lum O, Huang V, Adey M, et al. Development and validation of a geriatric depression screening scale: a preliminary report. J Psychiatr Res. 1982-1983;17(1):37-49. doi: 10.1016/0022-3956(82)90033-4

17. Araújo EC, Martins KP, Lima RJ, Costa KNFM. Concern with falls in elderly people attended in an Integral Attention Center. Rev Eletrônica Enferm. 2016;(1):1-9. doi: 10.5216/ree.v18.39899

18. Delbaere K, Close JCT, Mikolaizak AS, Sachdev PS, Brodaty H, Lord SR. The Falls Efficacy Scale International (FES-I). A comprehensive longitudinal validation study. Age Ageing. 2010 Mar;39(2):210-6. doi: 10.1093/ageing/afp225

19. Camargos FFO, Dias RC, Dias JMD, Freire MTF. Cross-cultural adaptation and evaluation of the psychometric properties of the Falls Efficacy Scale-International among elderly Brazilians (FES-IBRAZIL). Rev Bras Fisioter [Internet]. 2010 [cited 2021 Jan 31];14(3):237-43. Available from: https://www.scielo.br/j/rbfis/a/G6DXXwm9TS4zvFpyWxwnQPs/?format=pdf\&lang=en

20. Tomita Y, Arima K, Tsujimoto R, Kawashiri SY, Nishimura T, Mizukami S, et al. Prevalence of fear of falling and associated factors among Japanese community-dwelling older adults. Medicine (Baltimore). 2018;97(4):e9721. doi: 10.1097/MD.0000000000009721

21. Araújo IVS, Gomes NC, Nascimento JS, Ribeiro CCNR, Tavares DMS. Queda entre idosos: preditores e distribuição espacial. Rev Salud Pública. 2019;21(2):187-94. doi: 10.15446/rsap.v21n2.70298

22. Hoang OTT, Jullamate P, Piphatvanitcha N, Rosenberg E. Factors related to fear of falling among community-dwelling older adults. J Clin Nurs. 2017 Jan;26(1-2):68-76. doi: 10.1111/jocn.13337

23. Santos SCA, Figueiredo DMP. Preditores do medo de cair em idosos portugueses na comunidade: um estudo exploratório. Ciênc Saúde Colet. 2019;24(1):77-86. doi: 10.1590/1413-81232018241.29932016

24. Rosa BM, Abreu DPG, Santos SSC, Silva BT, Ilha S, Martins NFF. Associação entre risco de quedas e uso de medicamentos em pessoas idosas. Rev Baiana Enferm. 2017;31(4):e22410. doi: 10.18471/rbe.v31i4.22410

25. Reis KMC, Jesus CAC. Relationship of polypharmacy and polypathology with falls among institutionalized elderly. Texto Contexto Enferm. 2017;26(2):e03040015. doi: 10.1590/0104-07072017003040015

26. Faber LM, Scheicher ME, Soares E. Depressão, declínio cognitivo e polimedicação em idosos institucionalizados. Rev Kairós. 2017;20(2):195-210. doi: 10.23925/2176-901X.2017v20i2p195-210

27. Lee S, Oh E, Hong GRS. Comparison of factors associated with fear of falling between older adults with and without a fall history. Int J Environ Res Public Health. 2018 May;15(5):982. doi:10.3390/ijerph15050982

28. Ferreira MCG, Tura LFR, Silva RC, Ferreira MA. Social representations of older adults regarding 
quality of life. Rev Bras Enferm. 2017;70(4):806-13. doi: 10.1590/0034-7167-2017-0097

29. Lima IF, Azevedo RCS, Reiners AAO, Silva AMC, Souza LC, Almeida NA. Factors associated with the functional independence of elderly women in the city of Cuiabá. Rev Bras Geriatr Gerontol. 2016;19(5):827-37. doi: 10.1590/1809-98232016019.150231

30. Vogelsang EM. Older adult social participation and its relationship with health: rural-urban differences. Health Place. 2016;42:111-9. doi: 10.1016/j.healthplace.2016.09.010

Editora Científica: Tânia Solange Bosi de Souza Magnago

Editora Associada: Rafaela Andolhe

\section{Autor correspondente}

Katia Moreira da Silva

E-mail: katiakawam@hotmail.com

Endereço: Av. Maria Martins Fontoura, 1967, Granville I CEP: 78.731-226

\section{Contribuições de Autoria}

\section{1 - Kátia Moreira da Silva}

Concepção ou desenho do estudo/pesquisa; Análise e/ou interpretação dos dados; Revisão final com participação crítica e intelectual no manuscrito

\section{2 - Rosemeiry Capriata de Souza Azevedo}

Concepção ou desenho do estudo/pesquisa; Análise e/ou interpretação dos dados; Revisão final com participação crítica e intelectual no manuscrito

\section{3 - Annelita Almeida de Oliveira Reiners}

Concepção ou desenho do estudo/pesquisa; Análise e/ou interpretação dos dados; Revisão final com participação crítica e intelectual no manuscrito

\section{4 - Adriana Delmondes de Oliveira}

Concepção ou desenho do estudo/pesquisa; Análise e/ou interpretação dos dados; Revisão final com participação crítica e intelectual no manuscrito

\section{5 - Ageo Mário Cândido da Silva}

Concepção ou desenho do estudo/pesquisa; Análise e/ou interpretação dos dados; Revisão final com participação crítica e intelectual no manuscrito

\section{Como citar este artigo}

Silva KM, Azevedo RCS, Reiners AAO, Oliveira AD, Silva AMC. Prevalence and factors associated with fear of falling in elderly people with no history of falls. Rev. Enferm. UFSM. 2021 [Accessed on: Year Month Day]; vol.11 e80: 1-18. DOI: https://doi.org/10.5902/2179769264533 